\title{
Expression of Fusion Lytic Peptides Promotes Fungal Disease Resistance in Transgenic Plants
}

\author{
George S. Biliarski¹, Bakhyt Yertaeva², Amitava Mitra1* \\ ${ }^{1}$ University of Nebraska, Department of Plant Pathology, Lincoln, NE, USA \\ ${ }^{2}$ Institute of Plant Biology and Biotechnology, Almaty, Kazakhstan \\ Email: *amitra1@unl.edu
}

How to cite this paper: Biliarsji, G.S., Yertaeva, B. and Mitra, A. (2020) Expression of Fusion Lytic Peptides Promotes Fungal Disease Resistance in Transgenic Plants. American Journal of Plant Sciences, 11, 148-161.

https://doi.org/10.4236/ajps.2020.112012

Received: January 4, 2020

Accepted: February 15, 2020

Published: February 18, 2020

Copyright $\odot 2020$ by author(s) and Scientific Research Publishing Inc. This work is licensed under the Creative Commons Attribution International License (CC BY 4.0).

http://creativecommons.org/licenses/by/4.0/

(c) (i) Open Access

\begin{abstract}
Many organisms produce small proteins which exhibit antimicrobial activities. In recent decades, the biological role of antimicrobial peptides (AMP) has been recognized as the main factor in the defense mechanisms against a broad range of pathogenic microbes. The increased worldwide incidence of microbial resistance to antibiotics makes AMPs promising alternative for the control of microbial disease. Exploring the potential of AMPs in transgenic crops could lead to the development of new and improved cultivars which are resistant to various economically important diseases. In the present study, two fusion lytic peptide gene constructs coding for antimicrobial peptides were expressed in Nicotiana benthamiana tobacco plants and tested against three fungal pathogens, Sclerotinia sclerotiorum, Rhizoctonia solani, and $P y$ thium sp. Detached-leaf bioassay was employed for the transgenic plants carrying the fusion lytic peptide constructs (ORF13 and RSA1), transgenic vector only control plants (1234), and wild-type control plants (WT) against the three fungal pathogens. Symptom area of each leaf was measured with high accuracy and data were recorded and processed by statistical analyses. The results showed that transgenic plant lines ORF13 and RSL1 have substantial resistance to Sclerotinia sclerotiorum infection, producing significantly smaller lesion areas compared to vector only plant line 1234 and wild type plants. These transgenic lines also provided resistance against Rhizoctonia solani, however, these lines were not effective against the other fungal pathogen $P y$ thium sp.
\end{abstract}

\section{Keywords}

Antimicrobial Peptides, Antibiotic Resistance, Fungal Diseases, Plant Disease Control, Transgenic Plants

\section{Introduction}

Antimicrobial peptides (AMPs) are natural components of many living organ- 
isms' defense system. The common characteristic of these peptides is their ability to suppress a wide range of pathogenic microbes. AMPs are peptides which consist of up to 100 amino acids (AAs) [1]. In nature, AMPs are molecules with a high degree of biochemical and structural variability, which strongly correlates with the environmental diversity and the richness of living organisms. Despite their variability, AMPs share common characteristics such as positively charged AAs and the existence of hydrophobic or hydrophilic secondary structures [2]. The cationic nature of AMPs determines their ability to interact selectively with negatively charged microbial surfaces, which result in disruption or inhibition of microbial cells [3] [4] [5].

The fungal cell wall is a layered structure, with the inner layers performing a predominantly mechanical function, while the outer layers are associated with the physiological features of particular fungal species. In most species, the inner cell wall is composed of chitin with covalently attached branched $\beta-(1,3)$ glucans. This branched chitin is tied to proteins and polysaccharides which compose the outer cell wall, and their conformation varies with the fungal species [6]. AMPs interaction with the fungal cell wall varies depending on the AMPs type. Some AMPs disrupt the function and structure of the cell wall [7].

Diseases caused by microbial pathogen constitute a significant problem in crop production in the United States and worldwide. Pesticide application is a common practice to combat crop diseases. In the United States, over one billion pounds of agrochemicals are used annually for pest control, and the worldwide usage is approximately 5.6 billion pounds per year [8]. The systemic use of pesticides harms the environment and increases the risk of pesticide resistance incidents. Therefore, it is important that alternative means to combating crop diseases are developed and deployed.

Genetically engineering techniques have shown excellent results producing transgenic plants that inhibit plant pathogens. Successful commercial lines of genetically modified (GM) crops which are resistant to a broad range of pathogens have proven the effectiveness of this alternative approach. For example, experiments with model plants contained gene construct with AMPs coding sequences have been carried out by several laboratories. Results have shown the great capability of the AMPs genes constructs for protection against diseases caused by plant pathogens [9] [10].

In a previous study, mammalian lactoferrin cDNA was expressed in transgenic tobacco Nicotiana tabacum plants [9]. After inoculation with the bacterial pathogen Ralstonia solanacearum, most transgenic plants showed substantial delays of bacterial wilt symptoms. Further examples include investigations of the expression of the lactoferrin (LF) gene in transgenic tomato plants [10]. The plants were tested for resistance to the pathogen Ralstonia solanacearum that causes bacterial wilt in tomato. Susceptible tomato line F7926-96 was transformed by Agrobacterium transformation with modified LF cDNA and gene introgression confirmed by Southern blot. Additionally, lactoferrin expression was detected by northern and western blots. In $\mathrm{T} 1$ and $\mathrm{T} 2$ generations, kanamycin 
resistance was observed based on Mendelian segregation indicating a single locus insertion. Furthermore, Southern blot analysis showed a single corresponding band of LF cDNA in T1 and T2 kanamycin resistant plants. The two transgenic tomato lines inoculated with the pathogen $\left(1 \times 10^{7}\right.$ and $1 \times 10^{8}$ colony-forming units $(\mathrm{CFU} / \mathrm{mL})$ ) exhibited an early resistance and subsequent susceptibility. The survival rate until maturity was observed at $44 \%$ to $55 \%$ of the plants when they are inoculated with $1 \times 10^{5} \mathrm{CFU} / \mathrm{mL}$ of the pathogen. These results demonstrated the potential of the lactoferrin AMP gene for control of bacterial wilt of tomato [10].

Later, a bovine lactoferrin cDNA construct was used for transformation of wheat plants, and the transformants were inoculated with the head blight disease fungus Fusarium graminearum [11] [12]. Significant reduction of disease incidence was observed in the transgenic wheat plants, highly susceptible cultivar Bobwhite was resistant compared to control Bobwhite plants and two untransformed commercial wheat cultivars (Wheaton and ND 2710). The positive correlation between lactoferrin gene expression and the levels of disease resistance were demonstrated by an ELISA assay.

Expression of multiple genes in a single open reading frame (ORF) is an effective approach for combining desirable AMPs in a single cleavable polyprotein. This method is exploited from several animal Picornavirus and plant Potyviruses. Their polyproteins are processed co-translationally and post-translationally to mature forms by virus-encoded proteases [13] [14]. In an early experiment, the Foot-and-mouth disease virus (FMDV) 2A protein, which is responsible for the "cleavage" at its carboxyl-terminus, was used as cleavage sites of artificial polyproteins [13]. With cleavage efficiency ranging from $80 \%$ to $100 \%$, FMDV $2 \mathrm{~A}$ protease functioned properly and efficiently in plant cells. This work led the way of creating a single open reading frame of gene construct with multiple fused AMP peptides that can be used in transgenic plants for efficient protection against various plant pathogens.

In an effort to test effectiveness of $2 \mathrm{~A}$ protease mediated fusion constructs, two lactoferricin domain of lactoferrin together and two lactoferricin domains were fused with a full length lactoferrin with the FMDV 2A protease to generate multiple active functional AMP peptides. Transgenic tobacco plants were generated from these constructs and grown for several generations to obtain homozygous lines. In this work two selected lines were tested for resistance against three economically important fungal pathogens.

\section{Materials and Methods}

\subsection{Fusion Lytic Peptide Constructs and Transgenic Lines}

Previous laboratory research generated several fusion constructs by fusing synthetic or naturally occurring AMPs with lactoferrin. For this study, two fusion constructs, ORF13 and RSL1, were tested. ORF 13 was constructed by fusing two 44 amino acid long lactoferricin domains with an autocatalytic $2 \mathrm{~A}$ protease from 
the Foot and Mouth Disease virus [10]. Similarly, RSL1 had a lactoferrin gene in the middle flanked by two lactoferricin domains fused by the 2A protease [10] (Figure 1). A large number of transgenic tobacco lines expressing these constructs were obtained. Seeds from homozygous transgenic tobacco lines were used to obtain plants and to test them for fungal disease resistance.

\subsection{Preparation of Transgenic and Control Nicotiana benthamiana Plants}

In this study, four $N$. benthamiana lines were used; two transgenic lines containing lytic peptide genes ORF13 and RSA1 along with one empty vector transgenic line (1234) in the same binary construct, and one wild type control non-transgenic (WT) line. These lines were generated earlier via routine Agrobacterium-mediated transformation of $N$. benthamiana leaves using binary constructs ORF13 and RSL1. Seeds were sterilized by the following protocol: First seeds were placed in $2 \mathrm{ml}$ Eppendorf tubes. One milliliter of 10\% solution of commercial bleach $(\mathrm{NaOCl})$ was added to each tube. The seeds were immersed for five minutes. After removal of bleach from the tubes, the seeds were washed with distilled water three times. Washed seeds were immersed in $70 \%$ Ethanol for 30 seconds followed by three washes with distilled water. The distilled water was removed carefully from the Eppendorf tubes with a pipette.

The seeds were placed in magenta boxes on $50 \mathrm{ml}$ Murashige-Skoog (MS) solid plant media (Murashige-Skoog Plant Media: MS Salt 4.3 g, B1-Inositol (1 g Inositol, $10 \mathrm{mg}$ Thiamin $\mathrm{HCl}, 100 \mathrm{ml} \mathrm{ddH_{2 } \mathrm { O } )} 10 \mathrm{ml}$, Millers I (6 g K $\mathrm{K}_{2} \mathrm{PO}_{4}$,

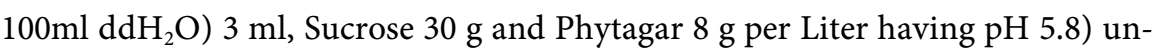
der aseptic condition in a Laminar Flow Clean bench. After placement on to the MS media, the seeds were streaked gently with a sterile glass loop. Antibiotic Kanamycin was added (100 mg per liter) to the MS media for transgenic plant seeds (ORF13, RSA1) and empty vector control (1234). No antibiotics were added in MS media for the wild type control (WT) plant seeds.

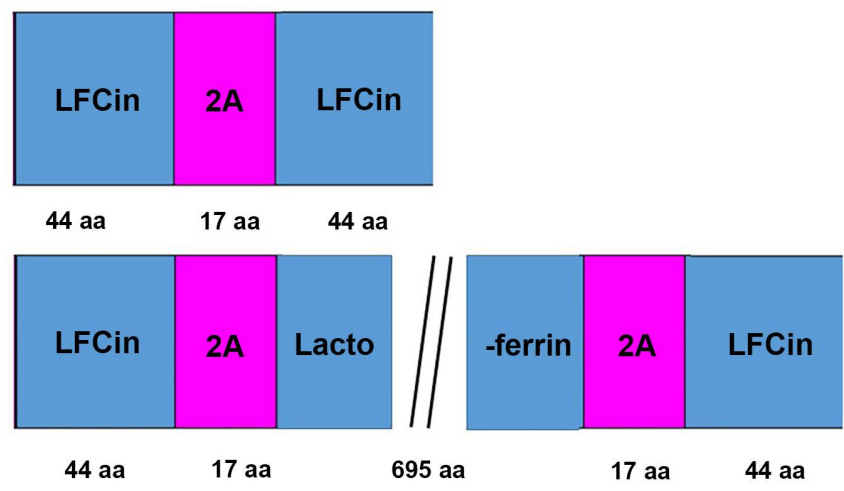

Figure 1. Fusion lytic protein constructs. Top: ORF13 consisting of two 44 amino acid long lactoferricin (LFCin) domain. Bottom: RSL1 consisting of a central 695 amino acid long lacoferrin gene flanked by two lactoferricin domains. Seventeen amino acid long $2 \mathrm{~A}$ protease peptide was obtained from the FMDV. 
Magenta boxes were placed in a plant growth chamber at $26^{\circ} \mathrm{C}$. The first germination was detected ten days after placing the seeds on MS media. The plants were kept in the magenta boxes for 30 days after germination.

After a month of growth in magenta boxes, the plants were transplanted in $500 \mathrm{~cm}^{3}$ pots filled with soil. Each pot was filled with approximately $400 \mathrm{~cm}^{3}$ steam-disinfected potting soil. A single plant was placed in each pot. Ten plants were prepared for each transgenic line and the wild type control. The pots were watered gently after planting. All plants were placed in a room with a steady temperature of $23^{\circ} \mathrm{C}, \sim 30 \%$ relative humidity, and artificial lighting with a day/night ratio of $14 \mathrm{~h} / 10 \mathrm{~h}$.

\subsection{Neomycin Phosphotransferase II Enzyme-Linked-Immunosorbent Assay (NPTII ELISA)}

Neomycin phosphotransferase II (NPTII) is a selectable marker gene used in the transgenic lines ORF13, RSA1, and empty vector control line 1234. Seven days after the transfer of seedlings to the pots, the presence of the NPTII protein product was qualitatively evaluated by an ELISA assay using an NPTII ELISA kit from Agdia, Inc. (52,642 Co. Rd. 1, Elkhart, IN 46514). Leaf biomass from each plant was placed in an Eppendorf tube. The biomass was ground in the tubes with the presence of $1 \times$ PEB1 extraction buffer in a 1:10 ratio (tissue weight in grams: buffer volume in milliliters). The assay was conducted in accordance with the instructions from the manufacturer.

\subsection{DNA Extraction from Plants}

The DNA from the transgenic plant lines ORF13, RSA1, 1234 empty vector control, and WT control was extracted by using the Thermo Fisher Plant DNAzol Reagent. For this procedure, the protocol provided by the manufacturer was used. The samples were ground using a mortar and pestle. After the pulverization, tissue was moved into Eppendorf tubes by using a sterile spatula. The Eppendorf tubes contained tissues were weighed on a scale as the scale was tared by the weight of an empty tube. $300 \mu \mathrm{l}$ plant DNAzol was added in each Eppendorf tube per $100 \mathrm{mg}$ plant tissue, mixed thoroughly and incubated for 5 minutes at $25^{\circ} \mathrm{C}$ by shaking. After incubation, $300 \mu \mathrm{l}$ of chloroform was added to each tube and mixed by vortex for approximately 30 seconds, then incubated for 5 minutes at $25^{\circ} \mathrm{C}$ with shaking, followed by centrifugation at $12000 \mathrm{RPM}$ at $4^{\circ} \mathrm{C}$ for 10 minutes. The supernatant was transferred into a fresh Eppendorf tube and mixed with $225 \mu$ of $100 \%$ ethanol for DNA precipitation. The samples were inverted eight times and then incubated at room temperature for 5 minutes. Subsequently, the tubes were centrifuged at 5000 RPM for four minutes, and the resulted supernatant was discarded and the DNA pellet retained. The DNA pellets were washed with $300 \mu \mathrm{l}$ Plant DNAzol-ethanol wash (mix of 1 volume DNAzol with 0.75 volume $100 \%$ ethanol) and the tubes were vortexed and incubated for five minutes, then centrifuged at 5000 RPM for 4 minutes and the supernatant was removed. The same washing step was repeated two more times. Following 
the washing steps, $300 \mu \mathrm{l}$ of $75 \%$ ethanol was added to the tubes, vortexed and then centrifuged at $5000 \mathrm{RPM}$ for 4 minutes. The supernatant was removed, and the tubes were oriented vertically for 5 minutes to remove the remaining ethanol with a micro-pipette. Finally, $70 \mu \mathrm{l}$ TE (Tris-EDTA) buffer was added in the tubes for dissolving DNA. The tubes were stored at $-80^{\circ} \mathrm{C}$ for further analysis.

\subsection{Neomycin Phosphotransferase II-Polymerase Chain Reaction (NPTII-PCR)}

Polymerase Chain Reaction (PCR) assay was performed for the transgenic plant lines ORF13, RSA1, 1234 empty vector control, and WT control, targeting Neomycin phosphotransferase II (NPTII) selectable marker gene. GoTaq ${ }^{\oplus}$ Green Master Mix was used following manufacturer's instructions. Two $\mu$ l DNA of each transgenic plant sample was added to $18 \mu \mathrm{l}$ reaction mixture containing $6 \mu \mathrm{l}$ $\mathrm{ddH}_{2} \mathrm{O}, 1 \mu \mathrm{l}$ Forward Primer, $1 \mu \mathrm{l}$ Reverse Primer along with $10 \mu \mathrm{GoTaq}^{\odot}$ Green Master Mix. The NPTII primers had the following sequence: Forward Primer; 221 5'-GAGGCTATTCGGCTATGAC-3', Reverse Primer 921

5'-ATCGGGAGCGGCGATACCG-3'. The thermal cycle was set to: 1) $94^{\circ} \mathrm{C}$ for 3 min., 2) $94^{\circ} \mathrm{C}$ for 30 sec., 3) $55^{\circ} \mathrm{C}$ for 40 sec., 4) $72^{\circ} \mathrm{C}$ for $1 \mathrm{~min} .40 \mathrm{sec}$., 5) $72^{\circ} \mathrm{C}$ for seven min., 6) $4^{\circ} \mathrm{C}$ forever, and steps two, three and four were repeated 35 times. The size of the amplified fragment of the tn5-NPTII gene was $700 \mathrm{bp}$.

\subsection{Lytic Peptide Gene-Polymerase Chain Reaction (PCR)}

Polymerase Chain Reaction (PCR) assay was performed for the transgenic plant lines ORF13 and RSA1 targeting lytic peptide genes using GoTaq ${ }^{\circledR}$ Green Master Mix solution and reaction buffers. Samples were prepared by using the protocol provided by the $\mathrm{GoTaq}^{\oplus}$ Green Master Mix manufacture and as stated in the paragraph above.

\subsection{Detached-Leaf Bioassay}

Detached-leaf bioassay was performed to screen the transgenic plants ORF13 and RSA1 carrying the lytic peptide genes, empty vector control plants 1234 , and wild type control (WT) plants against three fungal pathogens: Sclerotinia sclerotiorum, Rhizoctonia solani, Pythium sp. Rhizoctonia solani is a soil borne plant pathogenic basidiomycete fungus. It has a wide range of hosts. Rhizoctonia sola$n i$ attacking primarily the roots and lower stems of plants and causes serious plant losses especially during the seedling stage. Sclerotinia sclerotiorum is a plant pathogenic ascomycete fungus. It causes diseases known as white mold, cottony rot, soft rot or stem rot. Sclerotinia sclerotiorum also has a wide host range. The disease can spread very quickly from plant to plant in the field. It can even affect crops in the storage facilities after harvest. Common crops which are affected and suffer deleterious economic impacts are soybeans, cannola and peanuts. Pythium species are fungus-like eukaryotic microorganisms belonging to the parasitic oomycetes. Formally they were classified as fungi. Pythium cause diseases of economic importance such as root rots. The Pythium species used in 
this study is an aggressive isolate collected from western Nebraska in 2018. The isolate is currently being evaluated for species identification.

Each transgenic variety contained ten plants. For this experiment, two detached leaves per plant were used. Each bioassay had three replications per plant from each line. Sixty experiments for each plant lines per pathogen have been performed. The pathogens were grown in a petri dish on a solid Potato Dextrose Agar media (PDA) (39 g PDA powder to 1 liter $\mathrm{ddH}_{2} \mathrm{O}$ autoclaved on $120^{\circ} \mathrm{C}$ for 20 minutes). The PDA powder was procured from Sigma-Aldrich Company. Sclerotinia sclerotiorum and Rhizoctonia solani were grown for four days on the media before the experiment. Pythium sp. was grown for two days on the media before the experiment. By using a sterile glass tube, $8 \mathrm{~mm}$ plugs were cut in the media after the growth of the pathogen for use as inoculum. The detached leaves were placed in a plastic container $(40 \mathrm{~cm} \mathrm{~L} \times 31.8 \mathrm{~cm} \mathrm{~W} \times 6 \mathrm{~cm} \mathrm{H})$ with high humidity. The inoculation was performed by the use of sterile tweezers. Plugs were transferred to the leaves with the pathogen on the upper surface of the plug. The plugs' position on the leaves was approximately $15 \mathrm{~mm}$ down from the tip of each leaf. After the inoculation, containers were sealed with a transparent plastic wrap. Sealed containers were moved in a temperature-controlled room with an artificial light with a day/night ratio $14 \mathrm{~h} / 10 \mathrm{~h}$. The leaves inoculated with Sclerotinia sclerotiorum and Rhizoctonia solani were incubated for 5 days after inoculation. The Pythium sp. Inoculated leaves were incubated for 2 days after inoculation.

\subsection{Data Collection}

After incubation, leaves were photographed on a scaled photographic field. Each photo was processed with a graphics editor by marking the infected area of each leaf with a single color and the background color was changed to white. Compu Eye software was used [15] to measure the symptom area of each leaf quantitatively with high accuracy. The result of measuring the infected area as well as the total area was recorded in square millimeters.

\section{Results}

\subsection{Seeds Germination on Murashige-Skoog Solid Plant Media}

The wild type control Nicotiana benthamiana seeds were germinated in magenta boxes with $50 \mathrm{ml}$ solid Murashige-Skoog (MS) plant media. The transgenic plant seeds from lines ORF13, RSA1, and transgenic vector control were germinated on the same MS media supplemented with Kanamycin antibiotic. The first germination was observed ten days after the sowing. The plants were kept in the magenta boxes for 30 days after germination. During that period the plants were in good health and had an excellent growth rate with no signs of discoloration or fading. The transgenic lytic peptide lines and the transgenic control line grew on Kanamycin fortified MS solid media confirming that the plants carried the Neomycin phosphotransferase II marker gene and the linked lytic peptide genes. 


\subsection{Neomycin Phosphotransferase II Enzyme-Linked-Immunosorbent Assay (NPTII ELISA)}

The enzyme activity of NPTII selectable marker gene was evaluated qualitatively with an Enzyme-Linked-Immunosorbent Assay for the transgenic lines ORF13, RSA1, transgenic vector control and wild type (WT) control plants. This test was performed using Agdia NPTII (Neomycin phosphotransferase II) ELISA kit. All transgenic lines: ORF13, RSA1, empty vector control (1234), showed positive results for the existence of Neomycin phosphotransferase II marker gene in the plants' genome. According to the protocol, the appearance of blue color in the test wells is an indication for a positive result. Blue color was seen in the test wells of all the transgenic plant lines. No color was present in the test wells for the wild type control plants and the buffer negative control.

\subsection{Plant DNA Extraction}

DNA was extracted from the transgenic plant lines ORF13, RSA1, vector only control (1234) and WT control. The quality of extracted DNA was tested on $0.75 \%$ agarose gel $(750 \mathrm{mg}$ Agarose to $100 \mathrm{ml} \times 1$ Tris-Borate-EDTA buffer and $0.6 \mu \mathrm{l}$ of Ethidium Bromide solution $10 \mathrm{mg} / \mathrm{ml}$ ) electrophoresis was for $80 \mathrm{mi}$ nutes. The gel showed that the quality of the DNA was excellent.

\subsection{Polymerase Chain Reaction to Amplify Neomycin phosphotransferase II Selectable Marker Gene (NPTII-PCR)}

Polymerase Chain Reaction (PCR) assay was performed for the transgenic plant lines ORF13, RSA1 and vector control (1234) along with WT control, targeting the Neomycin phosphotransferase II (NPTII) selectable marker gene. All samples of the transgenic plants showed the expected fragment of the NPTII marker gene. The transgenic control plants which containing vector control (1234) also were positive for the NPTII marker gene. All samples were analyzed on $1.00 \%$ agarose gel (1000 mg Agarose to $100 \mathrm{ml} 1 \times$ Tris-Borate-EDTA buffer and $0.6 \mu \mathrm{l}$ of Ethidium Bromide solution $10 \mathrm{mg} / \mathrm{ml}$ ) electrophoresis at $100 \mathrm{~V}$ and $50 \mathrm{amps}$ was for 90 minutes. All NPTII positive samples showed a clear band at $700 \mathrm{bp}$ region on the agarose gel. All samples from the wild type $N$. benthamiana plants displayed negative results for the NPTII marker gene (Figure 2).

\subsection{Polymerase Chain Reaction to Amplify Lytic Peptide Genes}

Polymerase Chain Reaction (PCR) assays were employed for the transgenic plant lines ORF13 and RSA1. All kanamycin resistant transgenic plant lines also showed the targeted lytic peptide genes. The PCR products were confirmed with the agarose gel electrophoresis. For all transgenic plant line samples, clear bands were observed according to the expected amplicon size (Figure 3).

\subsection{Detached-Leaf Bioassay Experiment}

Detached-leaf bioassay was performed for the transgenic plants carrying fusion lytic peptide constructs ORF13, RSA1, transgenic vector control plants (1234), 


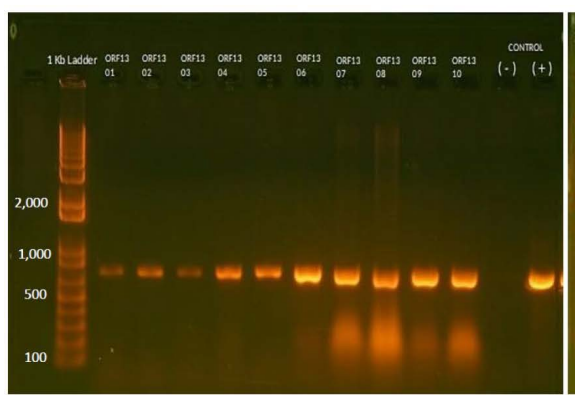

(a)

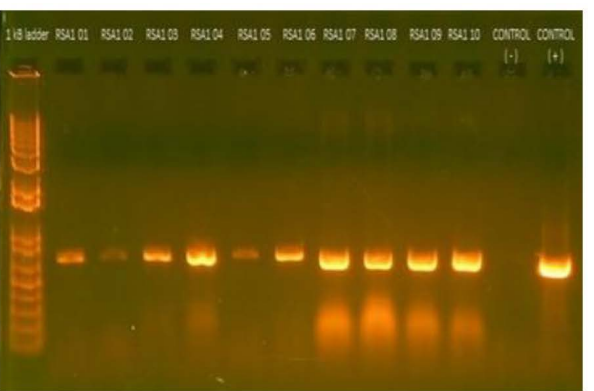

(b)

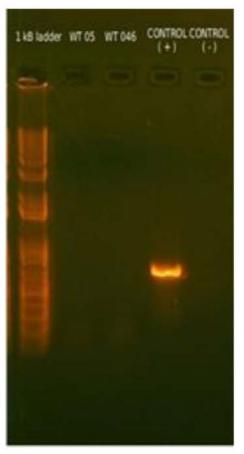

(c)

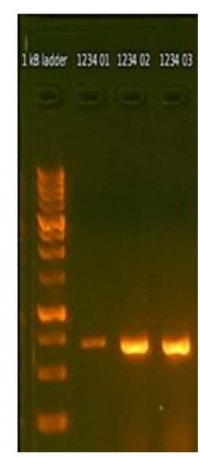

(d)

Figure 2. Agarose gel showing PCR amplification for NPTII gene in ORF 13 (a), RSL1 (b) and empty vector transgenic control 1234 (c) plants. All samples showed a clear band at 700 bp region on the agarose gel. PCR amplification using DNA from WT plants (d) did not show any bands. Lanes indicate the molecular size marker (100, 500, 1000 \& 2000 bp bands are indicated) and genomic DNA samples from independent transgenic lines of respective constructs.

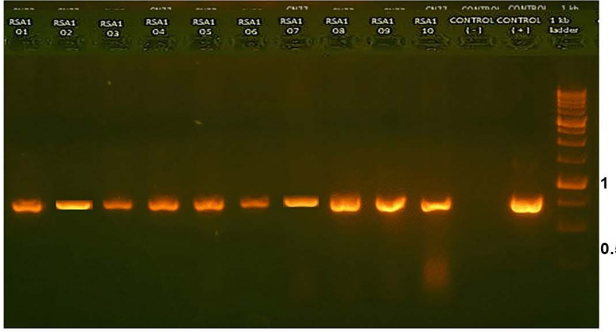

(a)

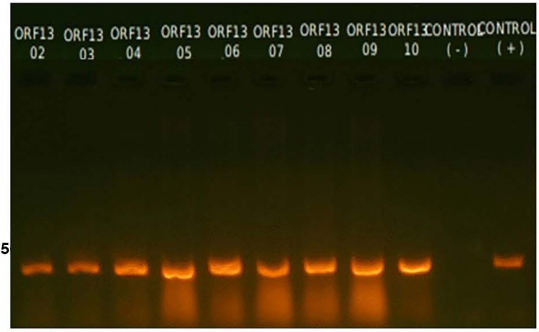

(b)

Figure 3. Agarose gels showing PCR amplification for RSA1 (a) and ORF13 (b) lytic peptide genes. The transgenic lines showed a band at $350 \mathrm{bp}$ for ORF13 and at $899 \mathrm{bp}$ for RSA1regions on the agarose gel. Lanes indicate the PCR amplification from different ORF13 and RSA1 transgenic lines along with the molecular size marker ( $1 \& 0.5 \mathrm{~kb}$ bands are indicated).

and wild control plants WT, against three fungal pathogens: Sclerotinia sclerotiorum, Rhizoctonia solani, and Pythium sp. The infected area of each leaf was measured, each individual measurement was recorded in square millimeters $\left(\mathrm{mm}^{2}\right)$.

\subsubsection{Experimental Design}

The goal of this experiment was to evaluate two transgenic lines ORF13, RSA1 
for resistance to three fungal plant pathogens Sclerotinia sclerotiorum, Rhizoctonia solani, and Pythium sp. compared to vector only control (1234) and WT control plants. Three fungal pathogens were tested sequentially for each plant. Three replications have been performed for each pathogen. Two leaves from each plant were inoculated per experiment generating 60 response values per tested pathogen. The experimental design is a completely randomized design (CRD), randomly assigning one plant variety to one pot.

\subsubsection{Inoculation of Detached Leaves with Sclerotinia sclerotiorum}

A total of 60 detached leaves were inoculated with S. sclerotiorum mycelial plugs and incubated for 5 days. At the end of the incubation period both the transgenic and WT control leaves showed between $90 \%-100 \%$ infections. The lesion areas on all the leaves were measured and statistically analyzed. Figure 4 shows lesions in four representative leaves and a bar diagram generated from statistical analysis.

Both the transgenic lines ORF13 and RSL1 showed significant resistance against $S$. sclerotiorum compared to the control plants, however, RSL1 lines more resistant than ORF13 lines. Comparing the differences of the mean, ORF1 and RSL1 transgenic lines have significant smaller symptom area than the wild type control. Hence, the transgenic plants demonstrated less susceptibility to the pathogen.

\subsubsection{Inoculation of Detached Leaves with Rhizoctonia solani}

A total of 60 detached leaves were inoculated with $R$. solani mycelial plugs and incubated for 5 days. At the end of the incubation period both the transgenic and WT control leaves showed between $85 \%-100 \%$ infections. The lesion areas on all the leaves were measured and statistically analyzed. Figure 5 shows lesions in four representative leaves and a bar diagram generated from statistical analysis.

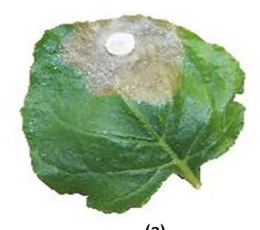

(a)

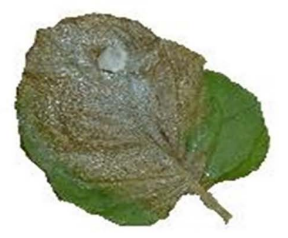

(c)

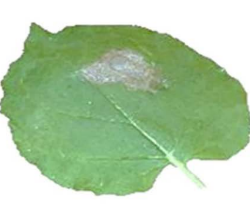

(b)

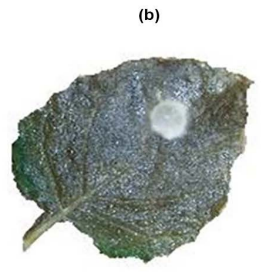

(d)

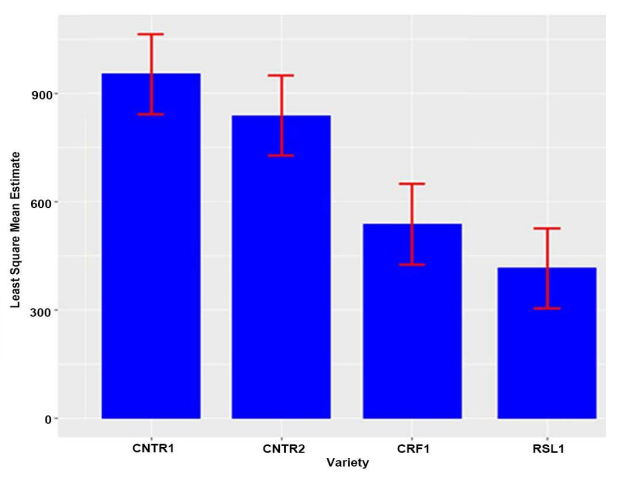

L1

Figure 4. Representative detached leaves of ORF13 and RSL1 inoculated with Sclerotinia sclerotiorum. (a) ORF13 leaf. (b) RSL1 leaf. (c) ORF13 leaf. (c) Wild Type leaf. (d) Vector only control (1234). The estimated Least Squares Means and the Standard Error for each variety are shown to the right. The blue bars indicate estimated Squares Means for the varieties and the red bars indicate standard error. In the graph CNTR1 is WT control, CNTR2 is vector only control and ORF1 is ORF13. 


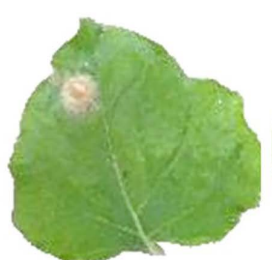

(a)

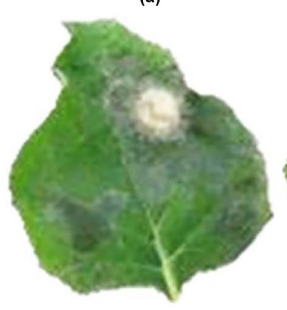

(c)

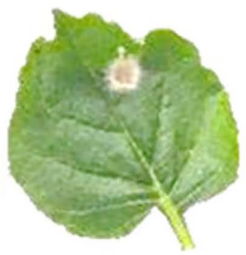

(b)

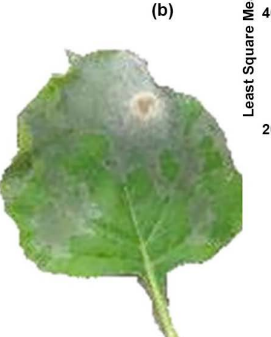

(d)

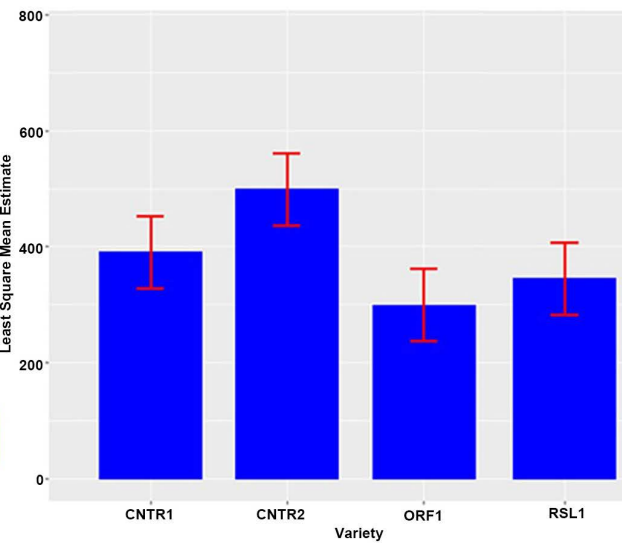

Figure 5. Representative detached leaves of ORF13 and RSL1 inoculated with Rhizoctonia solani. (a) ORF13 leaf. (b) RSL1 leaf. (c) ORF13 leaf (c) Wild Type leaf. (d) Vector control (1234). The estimated Least Squares Means and the Standard Error for each variety are shown to the right. The blue bars indicate estimated Squares Means for the varieties and the red bars indicate standard error. In the graph CNTR1 is WT control, CNTR2 is vector only control and ORF1 is ORF13.

Both the transgenic lines ORF13 and RSL1 showed enhanced resistance against Rhizoctonia solani compared to the control plants, however, ORF13 lines were more resistant than RSL1 lines. Overall, the level of resistance against Rhizoctonia solani was lower compared to $S$. sclerotiorum.

\subsubsection{Inoculation of Detached Leaves with Pythium sp.}

A total of 60 detached leaves were inoculated with Pythium sp. mycelial plugs and incubated for 2 days. At the end of the incubation period both the transgenic and WT control leaves showed $100 \%$ infections. The lesion areas on all the leaves were measured and statistically analyzed. Figure 6 shows lesions in four representative leaves and a bar diagram generated from statistical analysis.

The Pythium isolate used in this study turned out to be a super virulent and aggressive strain. The pathogen covered the entire control leaves and both the ORF13 and RSL1 leaves in just 2 days. Hence, both the ORF13 and RSL1 lytic peptide constructs were infective against the NE isolate of Pythium. The differences of the mean calculation indicate no significant smaller symptom area for the transgenic lytic peptide lines compared to the control lines.

\section{Discussion}

Many organisms produced small proteins which exhibit antimicrobial activities. The biological role of antimicrobial peptides (AMP) is being increasingly recognized as the main factor in the defense mechanisms against a broad range of pathogenic microbes [16]. The increased incidence of antimicrobial resistance worldwide due to overuse of antibiotics and pesticides makes AMPs promising alternatives for the treatment of microbial diseases. APMs are potential options 


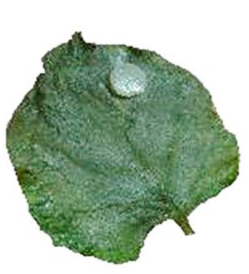

(a)

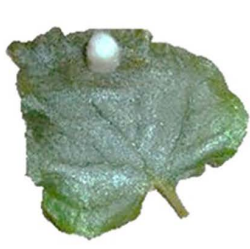

(c)

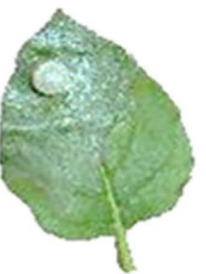

(b)

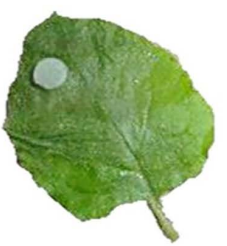

(d)

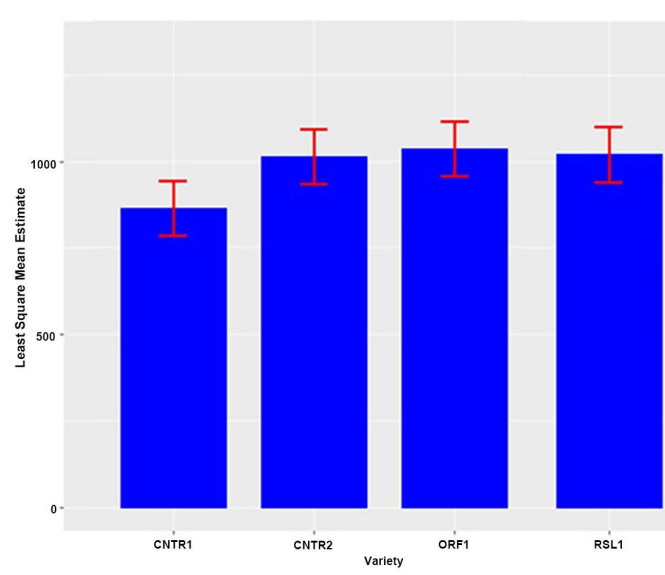

RsL1

Figure 6. Representative detached leaves of ORF13 and RSL1 inoculated with Pythium sp. (a) ORF13 leaf. (b) RSL1 leaf. (c) ORF13 leaf (c) Wild Type leaf. (d) Vector control (1234). The estimated Least Squares Means and the Standard Error for each variety are shown to the right. The blue bars indicate estimated Squares Means for the varieties and the red bars indicate standard error. In the graph CNTR1 is WT control, CNTR2 is vector only control and ORF1 is ORF13.

for use in agriculture for crop improvement and could lead to the development of new and improved cultivars that are resistant to various pathogenic diseases [17] [18] [19] [20].

In the present study, two lytic peptide gene constructs coding for antimicrobial peptides were expressed in tobacco plants and tested against three fungal pathogens, Sclerotinia sclerotiorum, Rhizoctonia solani, and Pythium sp. The resistance of tobacco transformants against these pathogens was examined in vivo with a detached leave bioassay system. Seeds of two homozygous transgenic $N$. benthamiana tobacco lines containing lytic peptide genes, one vector only control transgenic tobacco plant, and one wild type $N$. benthamiana tobacco plant were germinated on solid Murashige-Skoog (MS) plant media and later transferred to soil. The plants showed normal growth and development.

The DNA of transgenic lines ORF13 and RSA1 carrying non-plant multi-lytic peptide genes was tested with PCR using a specific primer for the respective genes. The presence of the lytic peptide genes was confirmed in all transgenic lines.

Our previous studies used transgenic detached leaves to establish effectiveness of non-plant and synthetic antimicrobial peptides against bacterial pathogens [9] [10]. In the current work, the detached leaf assay system was employed to test the effect of the two multi-lytic fusion peptide constructs on three fungal pathogens: Sclerotinia sclerotiorum, Rhizoctonia solani, and Pythium sp. The results showed that transgenic plant lines ORF13 and RSL1 have substantial resistance to Sclerotinia sclerotiorum infection by producing significantly smaller symptom area compared to control vector plant line 1234 and WT. The statistical analyzes confirmed the results of the bioassay. Estimated least square mean 
p-values for the transgenic lines RSL1 (0.0055) and ORF13 (0.0444) showed significant difference in the infected area comparing to the control WT. Similar result was observed against Rhizoctonia solani. The transgenic lines, however, were less effective against $R$. solani than $S$. sclerotiorum. The transgenic lines were not effective against Pythium sp., the assays did not show statistically significant smaller necrotic area compared to two controls. This could be due to the fact that Pythium is a primitive Oomycetes, not a true fungus and their coenocytic hyphal cell walls are primarily made of cellulose and $\beta-1,3$ glucan. Overall, these results confirmed earlier work from several laboratories demonstrating that transgenic expression of AMPs are effective in controlling many economically important plant diseases [16]-[20].

In conclusion, non-plant multi-lytic peptide constructs ORF13 and RSL1 confer resistance against two fungal pathogens Sclerotinia sclerotiorum and Rhizoctonia solani that cause economically important root rot and white mold diseases, respectively, in many crop plants. Hence, the use of a naturally occurring antimicrobial peptide lactoferricin fusion construct alone or in combination with the lactoferrin protein may provide broad-spectrum resistance against plant diseases.

\section{Acknowledgements}

The authors would like to thank Mahmoud H. Ghozlan and Serkan Tokgöz for their help with the experiments.

\section{Conflicts of Interest}

The authors state that the research was conducted in the absence of any commercial or financial relationships that could be construed as a potential conflict of interest.

\section{References}

[1] Singh, A., Phougat, N., Kumar, M. and Chhillar, A. (2013) Antifungal Proteins: Potent Candidate for Inhibition of Pathogenic Fungi. Current Bioactive Compounds, 9, 101-112. https://doi.org/10.2174/22115528112019990007

[2] Hegedüs, N. and Marx, F. (2013) Antifungal Proteins: More than Antimicrobials? Fungal Biology Reviews, 26, 132-145. https://doi.org/10.1016/j.fbr.2012.07.002

[3] Kaas, Q. (2010) Antimicrobial Peptides in Plants. In: Wang, G., Ed., Antimicrobial Peptides. Discovery, Design, and Novel Therapeutic Strategies, UNMC, NE, 40-71. https://doi.org/10.1079/9781845936570.0040

[4] Levy, O. (2004) Antimicrobial Proteins and Peptides: Anti-Infective Molecules of Mammalian Leukocytes. Journal of Leukocyte Biology, 76, 909-925. https://doi.org/10.1189/jlb.0604320

[5] Lee, D.W. and Kim, B.S. (2015) Antimicrobial Cyclic Peptides for Plant Disease Control. Plant Pathology Journal, 31, 1-11. https://doi.org/10.5423/PPJ.RW.08.2014.0074

[6] Latgé, J.-P. (2007) The Cell Wall: A Carbohydrate Armour for the Fungal Cell. Mo- 
lecular Microbiology, 66, 279-290. https://doi.org/10.1111/j.1365-2958.2007.05872.x

[7] Matejuk, A., Leng, Q., Begum, M.D., Woodle, M.C., Scaria, P., Chou, S.T. and Mixson, A.J. (2010) Peptide-Based Antifungal Therapies against Emerging Infections. Drugs Future, 35, 97-207. https://doi.org/10.1358/dof.2010.035.03.1452077

[8] Malmsten, M. (2014) Antimicrobial Peptides. Upsala Journal of Medical Sciences, 119, 199-204. https://doi.org/10.3109/03009734.2014.899278

[9] Zhang, Z., Coyne, D.P., Vidaver, A.K. and Mitra, A. (1998) Expression of Human Lactoferrin cDNA Confers Resistance to Ralstonia solanacearum in Transgenic Tobacco Plants. Phytopathology, 88, 730-734. https://doi.org/10.1094/PHYTO.1998.88.7.730

[10] Lee, T.-J., Coyne, D.P., Clemente, T.E. and Mitra, A. (2002) Partial Resistance to Bacterial Wilt in Transgenic Tomato Plants Expressing Antibacterial Lactoferrin Gene. Journal of the American Society for Horticultural Science, 127, 158-164. https://doi.org/10.21273/JASHS.127.2.158

[11] Han, J., Lakshman, D.K., Galvez, L., Mitra, S., Baenziger, P.S. and Mitra, A. (2012) Transgenic Expression of Lactoferrin Imparts Enhanced Resistance to Head Blight of Wheat Caused by Fusarium graminearum. BMC Plant Biology, 12, 33-39. https://doi.org/10.1186/1471-2229-12-33

[12] Trung., N.C., Lakshman, D.K., Han, J., Galvez, L.C. and Mitra, A. (2010) Transgenic Plants Expressing Antimicrobial Lactoferrin Protein Are Resistant to a Fungal Pathogen. Journal of Plant Molecular Biology and Biotechnology, 2, 1-8.

[13] Ma, C. and Mitra, A. (2002) Expressing Multiple Genes in a Single Open Reading Frame with the 2A Region of Foot-and-Mouth Disease Virus as a Linker. Molecular Breeding, 9, 191-199. https://doi.org/10.1023/A:1019760127368

[14] Riechmann, J.L., Sonia, L. and Juan, A.G. (1992) Highlights and Prospects of Potyvirus Molecular Biology. Journal of General Virology, 73, 1-16. https://doi.org/10.1099/0022-1317-73-1-1

[15] Bakr, E.M. (2005) A New Software for Measuring Leaf Area, and Area Damaged by Tetranychus urticae Koch. Journal of Applied Entomology, 129, 173-175. https://doi.org/10.1111/j.1439-0418.2005.00948.x

[16] Montesinos, E. (2007) Antimicrobial Peptides and Plant Disease Control. FEMS Microbiology Letter, 270, 1-11. https://doi.org/10.1111/j.1574-6968.2007.00683.x

[17] Goyal, R.K. and Mattoo, A.K. (2014) Multitasking Antimicrobial Peptides in Plant Development and Host Defense against Biotic/Abiotic Stress. Plant Science, 228, 135-149. https://doi.org/10.1016/j.plantsci.2014.05.012

[18] Jung, Y.J., Lee, S.Y., Moon, Y.S. and Kang, K.K. (2012) Enhanced Resistance to Bacterial and Fungal Pathogens by Overexpression of a Human Cathelicidin Antimicrobial Peptide (hCAP18/LL-37) in Chinese Cabbage. Plant Biotechnology Reports, 6, 39-46. https://doi.org/10.1007/s11816-011-0193-0

[19] Ramadevi, R., Rao, K.V. and Reddy, V.D. (2011) Antimicrobial Peptides and Production of Disease Resistant Transgenic Plants. In: Vudem, D.R., Poduri, N.R. and Khareedu, V.R., Eds., Pests and Pathogens: Management Strategies, CRC Press, Taylor \& Francis Group, New York, 379-452.

[20] Osusky, M., Zhou, G., Osuka, L., Hancock, R.E., Kay, W.W. and Misra, S. (2000) Transgenic Plants Expressing Cationic Peptide Chimeras Exhibit Broad-Spectrum Resistance to Phytopathogens. Nature Biotechnology, 18, 1162-1166.

https://doi.org/10.1038/81145 